\title{
Konsolidierende Bestrahlung beim Hodgkin-Lymphom wichtig
}

Ein frühes Hodgkin-Lymphom $(\mathrm{HL})$ wird initial mit einer Kombinationschemotherapie behandelt. Empfohlen wird eine anschließende konsolidierende Strahlentherapie (RT), die jedoch nicht immer durchgeführt wird. Zu Unrecht, wie eine retrospektive Analyse zeigt.

D as Team um Rahul R. Parikh wertete Daten der US-amerikanischen $\mathrm{Na}$ tional Cancer Database (NCDB) aus. Bei den Patienten war zwischen 1998 und 2011 die Diagnose HL im Stadium I/II gestellt worden. Ziel war es, eine Assoziation zwischen Strahlentherapie, Kovariablen und dem Behandlungsergebnis zu finden. Insgesamt gingen Daten von 29.752 Patienten (Alter: median 37 Jahre) in die Analyse ein. Alle Patienten hatten primär eine Chemotherapie erhalten. Für 17.170 Patienten lagen Daten zum Gesamtüberleben (OS) vor.

$54 \%$ der Patienten hatten nach der Chemotherapie eine RT mit median 30,6 Gy erhalten. Ihre Anwendung als Kon- solidierungstherapie war signifikant assoziiert mit einem jüngeren Alter $(\leq 40$ Jahre), einem günstigen Versicherungsstatus, einem höheren sozioökonomischen Status sowie der Behandlung in einem spezialisierten regionalen Krebszentrum ( $\mathrm{p}<0,05$ für alle Faktoren).

Die Rate des 5-Jahres-OS lag bei Patienten, die eine RT erhalten hatten, bei $94,5 \%$, bei denjenigen ohne diese Konsolidierungstherapie bei 88,9\% ( $p<0,01)$. Die RT war ein signifikanter Prädiktor für das Überleben in der As-treated- (Hazard Ratio [HR] 0,53; 95\%-Konfidenzintervall [95\%-KI] 0,49-0,58; p < 0,01) sowie in der Intention-to-treat-Analyse $(\mathrm{p}<0,01)$. Auch nach statistischer Be- rücksichtigung klinischer und pathologischer Einflussfaktoren blieb die RT-Konsolidierung mit einem verbesserten OS assoziiert (HR 0,46; 95\%-KI 0,38-0,56; $\mathrm{p}<0,01)$. Die Anwendung der RT-Konsolidierung war jedoch im Beobachtungszeitraum von $55 \%$ auf $44 \%$ gesunken. Meist war sie schon initial im Behandlungsplan nicht berücksichtigt worden.

Fazit: Dieser Analyse zufolge ist die Konsolidierungs-RT beim frühen HL klar mit einem besseren Überleben verbunden. Die Forscher mahnen daher einen besseren Zugang der Patienten zu dieser Therapie an. Der Verzicht darauf scheine häufig aus nicht medizinischen Gründen zu erfolgen.

Friederike Klein

Parikh RR et al. Early-stage classical Hodgkin lymphoma: The utilization of radiation therapy and its impact on overall survival. Int J Radiat Oncol Biol Phys. 2015;93(3):684-693.

\section{Mit i-FDG-PET verlässliche Hodgkin-Prognose}

\section{Mit einer Positronenemissionstomografie mittels Fluorodeoxyglukose (FDG-PET) lassen sich verbliebene Tumorzellen nachweisen. Beim fortge- schrittenen Hodgkin-Lymphom ist das ein Hinweis auf ein drohendes Rezidiv. Nun wurde der prognostische Wert dieser Methode für das Frühstadium untersucht.}

W eil Hodgkin-Patienten lange überleben, bekommen Spätfolgen der Therapie große Bedeutung. In fortgeschrittenen Stadien ist ein zwischen verschiedenen Therapieschritten (interim, i) vorgenommenes FDG-PET von prognostischem Wert: Es macht folgenreiche Behandlungen bei Patienten mit besonders guter Prognose überflüssig und bietet die Basis dafür, die Therapie bei schlechter Prognose zu intensivieren.

Für das Frühstadium initiierten italienische Kollegen eine retrospektive multizentrische Studie, in der alle Patienten mit einer Chemotherapie aus Adriamycin, Bleomycin, Vinblastin und Dacarbazin (ABVD) sowie Bestrahlung behandelt worden waren. 257 Patienten erhielten ein FDG-PET bei Diagnose sowie nach den ersten zwei ABVD-Zyklen. Alle Scans wurden verblindet mithilfe des Deauville-5-Punkte-Scoring-Systems begutachtet. Es wurden zwei Cutoff-Werte

Tab. 1: i-FDG-PET und Überleben

\begin{tabular}{|c|c|c|}
\hline & \multicolumn{2}{|c|}{ i-FDG-PET } \\
\hline & negativ & positiv \\
\hline 5-Jahres-PFS (DC 3) & $98,1 \%$ & $83,7 \%$ \\
\hline 5-Jahres-PFS (DC 4) & $97,7 \%$ & $78,6 \%$ \\
\hline 5-Jahres-OS (DC 3) & $98,5 \%$ & $93,0 \%$ \\
\hline 5-Jahres-OS (DC 4) & $98,6 \%$ & $89,3 \%$ \\
\hline \multicolumn{3}{|c|}{$\begin{array}{l}i \text {-FDG-PET = interim Positronenemissionstomografie } \\
\text { mittels Fluorodeoxyglukose; } P F S=\text { progressionsfreie } \\
\text { Überleben; OS = Gesamtüberleben; } D C=\text { Deauville- } \\
\text { Cutoff }\end{array}$} \\
\hline
\end{tabular}

analysiert: ein Deauville-Score $\geq 3$ bzw. $\geq 4$. Bei einem medianen Follow-up von 56 Monaten lagen die Raten für 5-JahresGesamtüberleben (OS), krankheitsspezifisches bzw. progressionsfreies Überleben (PFS) für die gesamte Kohorte bei 97,5\%, 98,3\% bzw. 95,6\%. 43 der 257 Patienten $(16,7 \%)$ hatten ein positives i-FDG-PET (Deauville-Score 3-5). Die Raten für 5-Jahres-PFS und 5-Jahres-OS für die iFDG-PET-negativen bzw. -positiven Patienten sind in Tab. 1 aufgeführt.

Fazit: Ein FDG-PET nach zwei Zyklen ABVD ist bei Hodgkin-Patienten im Frühstadium prognostisch für die Vorhersage von PFS und Gesamtüberleben. Ein negatives i-FDG-PET bedeutet ein niedriges Rezidivrisiko. Die Fortführung der Therapie bei positivem i-FDGPET ermöglicht meist eine dauerhafte Komplettremission. Christina Berndt

Simontacchi G et al. Interim PET After Two ABVD Cycles in Early-Stage Hodgkin Lymphoma: Outcomes Following the Continuation of Chemotherapy Plus Radiotherapy. Int J Radiat Oncol Biol Phys. 2015;92(5):1077-83. 